\title{
AGILE Observations of Variable and transient $\gamma$-ray sources in the Galactic plane, Catalog improvements
}

\author{
Andrew Chen ${ }^{* \dagger}$ \\ INAF - IASF Milano \\ E-mail: chen@iasf-milano.inaf.it
}

AGILE has provided continuous monitoring of the Galactic plane in $\gamma$-rays in more than three years of operation [1]. Thanks to its sensitivity at energies near $100 \mathrm{MeV}$, AGILE has observed variability and transient behavior in a number of sources. Simultaneous hard X-ray coverage, rapid alerts to the astronomical community, and multiwavelength campaigns have provided identifications for some of these sources and placed constraints on others. We provide an overview of these observations and their possible counterparts, including microquasars and colliding wind binaries, as well as work in progress on a catalog of AGILE variable and transient sources.

25th Texas Symposium on Relativistic Astrophysics

December 6-10, 2010

Heidelberg, Germany

\footnotetext{
*Speaker.

$\dagger$ On behalf of the AGILE Galactic Sources Working Group.
} 


\section{Introduction}

AGILE (Astro-rivelatore Gamma a Immagini LEggero), an Italian Space Agency (ASI) highenergy astrophysics mission [1], was launched on April 23, 2007 and has been observing the gamma-ray sky since July 2009 [2]. The satellite contains co-aligned detectors, the $30 \mathrm{MeV}$ $50 \mathrm{GeV}$ Gamma-Ray Imaging Detector, GRID [3, 4] and the 18-60 keV hard X-ray detector SuperAGILE [5].

The wide field of view of these instruments ( $2.5 \mathrm{sr}$ for the GRID and $1 \mathrm{sr}$ for Super-AGILE ) have allowed AGILE to continuously monitor the Galactic plane for variability and transient events. During this period, AGILE performed its observations in pointing mode. During each observation period, lasting on average about three weeks, AGILE observed about one fifth of the sky while providing the sources within the field of view a source live time fraction of approximately 0.5 , taking into account Earth occultation and passages through the South Atlantic Anomaly. Over the course of one day, a typical source at $30^{\circ}$ off-axis would receive an exposure of $\approx 2 \times 10^{7} \mathrm{~cm}^{2} \mathrm{sec}$ at $100 \mathrm{MeV}$. This compares favorably to the one-day exposure at that energy provided by Fermi , which has a much higher sensitivity $\left(>3 \times 10^{8} \mathrm{~cm}^{2} \mathrm{sec}\right)$ at $\mathrm{GeV}$ energies.

Monitoring variability and transient events in the Galactic plane is considerably more difficult than the relatively straightforward observation of extragalactic transients. The much greater density of gamma-ray sources and possible source counterparts, and their clustering in star-forming regions and along the tangents of the Galactic spiral arms, provides ample opportunity for source confusion. The Galactic diffuse emission is both bright and varies spatially on angular scales comparable to the gamma-ray point source dispersion, requiring accurate and detailed modeling [6]. High particle background and low statistics at short timescales require sophisticated background rejection techniques [7] and robust automatic quicklook capability [8]. Nevertheless, AGILE has been able to provide rapid alerts to the astronomical community in response to gamma-ray transient events, resulting in coverage at radio, optical, and X-ray wavelengths.

Gamma ray transients are most often seen on the time scale of 1-2 days, with luminosities equivalent to a few $\times 10^{34} d_{\mathrm{kpc}}^{2}$ erg/s. Both simultaneous coverage with Super-AGILE and alerts to other X-ray telescopes have confirmed that, by and large, Galactic gamma-ray transient events are not associated with outbursts in hard X-rays, constraining models of microquasar emission as a possible source of gamma-ray transient activity. Furthermore, in most cases, no obvious X-ray source candidates have been found. Exceptions include microquasars such as Cygnus X-3 and $\mathrm{LSI}+61^{\circ} 303$ and colliding wind binaries such as Eta Carinae and are discussed below. Variability of the Crab pulsar wind nebula [9] is discussed elsewhere in these proceedings.

\section{Gamma-ray data analysis procedures}

AGILE monitoring of $\gamma$-ray variable and transient events is performed using a number of automated scripts based on the AGILE -GRID software package, a subset of which is publicly available at the ASI Data Center web site (http://agile.asdc.asi.it/).

Only events flagged as confirmed $\gamma$-ray events ( $\mathrm{G}$ class events, corresponding to an on-axis effective area of $\sim 350 \mathrm{~cm}^{2}$ at $100 \mathrm{MeV}$ [2]) by the FM3.119 particle background filter are used. The AGILE photon counts, exposure, and Galactic background maps are generated with a bin size 
of $0.2^{\circ} \times 0.2^{\circ}$ for energies greater than $100 \mathrm{MeV}$. The analysis is performed over a region of $10^{\circ}$ radius.

The multi-source likelihood analysis method is used. A map of significance is produced, defining regions of analysis corresponding to high-significance spots and continuously monitored positions. The position and flux of all the sources in each analysis region are optimized iteratively, taking into account the Galactic diffuse $\gamma$-ray radiation and isotropic emission [6]. This method evaluates the statistical significance of source detection and determines fluxes and position of the point sources included in the source model. The statistical significance is determined in terms of the log-likelihood Test Statistic (TS) defined as in [10], asymptotically distributed as a $\chi^{2}$ for 3 degrees of freedom $\left(\chi_{3}^{2}\right)$ [11] corresponding to the source flux, galactic longitude and latitude.

The automated search procedure is divided in two steps. In the first step, an automated search analysis is performed over intervals of 1 day for pointing mode and 2 days for spinning mode. Both discrete intervals with fixed boundaries and continuously overlapping intervals with 1-hour offsets are analysed. In the second step, the significant detections $(T S \geq 9)$ from the first step are selected for further analysis. In cases with time-overlapped detections, the detection with the largest value of the TS parameter is selected as the flare peak. For this subclass of selected flare peaks, additional manual analysis is performed [12].

The multi-source likelihood method is very efficient for relatively strong sources, providing the pre-trial statistical significance which must be converted to a post-trial probability when used in repeated systematic searches. An independent method which takes into account multiple comparison corrections, was developed [11] based on the False Discovery Rate technique (FDR, [13 - 15]). The FDR method is designed to control the expected rate of false detections due to statistical fluctuations when performing repeated trials. We apply this approach to the AGILE data by performing a blind search for (persistent or transient) sources in large daily counts maps of the Galactic plane. The null hypothesis for these daily maps is the (background dominated) counts distribution of the Galactic plane as a whole. The random fluctuations and the diffuse gamma-ray emission of these daily maps are well described by Poissonian distributions in AGILE -GRID data. Candidate sources in the daily maps are identified as significant deviations from the total distribution of counts from that particular day. The FDR method is used also at the source level. In this case the search is optimized for flaring episodes in the counts light curve extracted from the position of a single candidate source location. In the (verified) assumption that the average source flux at a given position is typically below the instrument sensitivity, unless it is producing (rare) flares, the null hypothesis in this case is obtained by measuring the distribution of photon counts for the specific sky location observed at intervals of one day.

\section{Variable Galactic Sources}

The Galaxy hosts a large number of high-energy $\gamma$-ray sources. Radiation in this energy range provides a wealth of information on relativistic phenomena near compact objects, as well as on the generation, propagation and interaction of high energy cosmic rays. Several source classes have been recently identified by ground-based and satellite gamma-ray detectors. They include massive molecular clouds, accreting compact objects, rotation-powered neutron stars and their wind nebulae, massive binary systems, remnants of supernova explosions, Galactic candidate jet sources 
and colliding winds sources in close systems. More details on the association of point-like $\gamma$-ray sources are provided in the first AGILE [2] and Fermi -LAT [16] catalogs. The identification of a source class is often based on temporal evolution and coordinated multiwavelength observations. AGILE has provided the alerts of transient gamma-ray emission from several source candidates. In some cases a clear source identification was provided by means of correlated studies.

\subsection{Cygnus X-3}

In addition to detecting weak persistent emission from a $\gamma$-ray source spatially compatible with the position of the microquasar X-ray binary Cygnus X-3, an exceptional X-ray binary which sporadically produces powerful radio jets, AGILE reported the detection of transient gamma-ray emission from the source. (see [17] and references therein). The region around Cygnus X-3 is complex, hosting star formation sites, OB associations and several prominent $\gamma$-ray sources. The angular resolution of AGILE satisfactorily resolves the field surrounding Cyg X-3 at gamma-ray energies, allowing it to be distinguished from the steady gamma-ray pulsar 1AGL J2032+4102/0FGL $\mathrm{J} 2032.2+4122$, which is $0^{\circ} .4$ away from Cyg X-3. Integrating all AGILE data, we find a weak (4.6 sigma) gamma-ray source consistent with the Cyg X-3 position with flux compatible with that reported by the Fermi -LAT catalog [16]. Four gamma-ray flares (each lasting 1-2 days) were detected by the AGILE satellite simultaneously with special spectral states of Cygnus X-3 during the period mid-2007/mid-2009. Continuous monitoring of these sources allowed us to detect other flaring episodes [12]. The statistical significance of all flares was individually assessed by both the maximum likelihood analysis and False Discovery Rate method. These flares are all associated with particular Cyg X-3 radio and X-ray/hard X-ray states. Remarkably, all gamma-ray flares occur during distinct minima of the hard X-ray lightcurve. either during soft X-ray states or their transitions to or from quenched hard X-ray states. Moreover we found that 3 out of 4 gamma-ray flares are distinctively produced before major radio flares. Our results show clearly that the flaring gamma-ray emission occurs only at special transitional states, which are associated with bright soft $\mathrm{X}$-ray states and/or the very low radio emission which precedes major radio flares [17]. Gamma-ray emission at these energies would imply Lorentz factors of $>10^{5}$ for leptonic emission models and $>10^{2}$ for hadronic scenarios. Fermi -LAT obtained consistent results and independently identified Cyg X-3 as a variable gamma-ray source, through the detection of its orbital period [18].

\subsection{Cygnus X-1}

AGILE reported the main results of an extensive search for transient gamma-ray emission from Cygnus X-1 (Cyg X-1), a black hole binary system, carried out in the energy range from $100 \mathrm{MeV}$ to $3 \mathrm{GeV}$, during the period 2007 July - 2009 October [19]. During the observation period the source was in the "hard" X-ray spectral state. AGILE reported an episode of significant transient gamma-ray emission detected on 2009, October 16 in a position compatible with the Cyg X-1 optical position. This episode, which occurred during a hard spectral state of Cyg X-1, showed that a 1-2 day time-variable emission above $100 \mathrm{MeV}$ can be produced during hard spectral states, having important theoretical implications for current Comptonization models for Cyg X-1 and other microquasars. Another flaring episode was detected by AGILE from 2010, June 30 to 2010, July 2 [20]. Aside from these flaring episodes, no significant persistent gamma-ray emission was detected by AGILE [19]. By integrating all available data we obtain a $2 \sigma$ upper limit for the total 
integrated flux of $3 \times 10^{-8} \mathrm{ph} \mathrm{cm}^{-2} \mathrm{~s}^{-1}$ in the energy range $100 \mathrm{MeV}-3 \mathrm{GeV}[11,19]$. The reported flaring episodes are not confirmed so far by Fermi -LAT .

\subsection{Gamma-Cygni region}

Another variable source reported by AGILE et al. is 1AGL J2022+4032, coincident with the interior of the radio shell of the supernova remnant Gamma Cygni (SNR G78.2+2.1) and its Pulsar Wind Nebula in the Cygnus Region. The source has been identified by Fermi -LAT as a $\gamma$-ray pulsar, LAT PSR J2021+4026 [21]. AGILE presented long-term observations of 1AGL J2022+4032 showing that the flux variability of 1AGL J2022+4032 appears to be greater than the level predicted from statistical and systematic effects. We evaluated the possibility that the $\gamma$-ray emission may be due to the superposition of two or more point sources, some of which may be variable, considering a number of possible counterparts. Although we determined that the most probable counterpart would be an unidentified X-ray quiet microquasar [22], recent detection of variability in the Crab pulsar[9, 23] makes a variable pulsar wind nebula hypothesis more likely, while a background blazar remains unlikely.

\subsection{LSI $+61^{\circ} 303$}

Another variable source seen by AGILE is coincident with the microquasar $\mathrm{LSI}+61^{\circ} 303$. In July-August 2009, the gamma-ray flux for $E>100 \mathrm{MeV}$ increased by at least a factor of four above its average flux. Fermi $-L A T$ positively identified the $\gamma$-ray source with LSI $+61^{\circ} 303$ by detecting variability of the $\gamma$-ray flux consistent with the binary orbital period [24]. Curiously, the phases of the peaks of the $\gamma$-ray emission observed by AGILE are not particularly concentrated around the peaks of the orbital modulation found by Fermi, although a high fraction lie near the peak observed in the TeV emission by MAGIC [25].

\subsection{Eta Carinae}

Extensive observations by the $\gamma$-ray AGILE satellite of the Galactic region hosting the Carina nebula and the remarkable colliding wind binary Eta Carinae during the period 2007 July -2009 January were reported in [26]. AGILE detected a gamma-ray source (1 AGL J1043-5931) consistent with the position of $\eta$-Car. If 1AGL J1043-5931 is indeed associated with the Carina system, the AGILE data provide the first detection above $100 \mathrm{MeV}$ of a colliding wind binary. A 2-day $\gamma$-ray flaring episode of 1AGL J1043-5931 on 2008 Oct. 11-13 possibly related to a transient acceleration and radiation episode of the strongly variable shock in the system was also reported [26]. The steady emission from this source was also detected by Fermi -LAT [27].

\section{Conclusion}

AGILE has been observing the Galactic plane since the beginning of its mission. The AGILE -GRID is well suitable for such searches due to several reasons. It has a large field of view and its sensitivity is optimized in the $\sim 100 \mathrm{MeV}$ range. Fast data transmission and processing allow fast alerts to be provided to the community via a dedicated automated process with various statistical tools and a scientific team devoted particularly to the search for gamma-ray transient events. By 
monitoring the Galactic plane in both gamma-rays and X-rays continuously for two years, AGILE has been able to observe a number of Galactic gamma-ray transients and variable sources, providing fast alerts to the astronomical community for simultaneous observations at multiple wavelengths. AGILE has discovered that Galactic transient events tend to occur on short time scales and energies below $400 \mathrm{MeV}$, with no correlated emission in hard X-rays and rarely having probable X-ray counterparts. AGILE has revealed correlated gamma-ray emission from Cygnus X-3, variability of a source coincident with Fermi pulsar J2021+4026, and extreme variability in microquasar LSI $+61^{\circ} 303$. The sensitivity of AGILE to gamma-rays below $400 \mathrm{MeV}$ and simultaneous hard X-ray coverage will continue to provide useful monitoring of Galactic gamma-ray variability and transient events in the Fermi era. A comprehensive survey of AGILE gamma-ray transients and variable sources is in preparation [28].

\section{Acknowledgments}

The AGILE Mission is funded by the Italian Space Agency (ASI) with scientific and programmatic participation by the Italian Institute of Astrophysics (INAF) and the Italian Institute of Nuclear Physics (INFN).

\section{References}

[1] M. Tavani, et al., Astronomy and Astrophysics 502 (2009) 995.

[2] C. Pittori et al., Astronomy and Astrophysics 506 (2009) 1563.

[3] G. Barbiellini et al., Nucl. Inst. Meth. A 490 (2002) 14.

[4] M. Prest et al., Nucl. Inst. Meth. A 501 (2003) 280.

[5] M. Feroci et al., Nucl. Inst. Meth. A 581 (2007) 728.

[6] A. Giuliani et al., Mem. Soc. Astron. It. Suppl. 5 (2004) 135.

[7] A. Giuliani et al., Nucl. Inst. Meth. A 568 (2006) 692.

[8] A. Bulgarelli et al., AGILE-GRID Automated Web-based Analysis System for Fast Detection of Gamma-ray Transients in proceedings of Astronomical Data Analysis Software and Systems XVIII, Quebec, Canada, ASP Conf. Ser. 411 (2008) 362.

[9] M. Tavani, et al., Science 331 (2011) 736.

[10] J.R. Mattox et al., The Astrophysical Journal 461 (1996) 396.

[11] S. Sabatini et. al., The Astrophysical Journal Letters 712 (2010) L10.

[12] A. Bulgarelli et al., Astronomy and Astrophysics (2011), submitted.

[13] Y. Benjamini \& Y. Hochberg, J. R. Stat. Soc. B 57 (1995) 289.

[14] C. J. Miller et al. The Astrophysical Journal 122 (2001) 349.

[15] A. M. Hopkins et al., The Astronomical Journal 123 (2002) 1086.

[16] A. A. Abdo et al., The Astrophysical Journal Supplement Series 188 (2010) 405.

[17] M. Tavani et al., Nature 462 (2010) 620. 
[18] A. A. Abdo et al., Science 326 (2009) 1512.

[19] E. Del Monte et al., Astronomy and Astrophysics 520 (2010) A67.

[20] S. Sabatini et al., The Astronomers Telegram 2715 (2010).

[21] A. A. Abdo et al., Science 325 (2009) 840.

[22] A. W. Chen et al., Astronomy and Astrophysics 525 (2011) A33.

[23] A. A. Abdo et al., Science 331 (2009) 739.

[24] A. A. Abdo et al., The Astronomical Journal Letters 701 (2009)123.

[25] J. Albert et al., Science 312 (2006) 1771.

[26] M. Tavani et al., The Astrophysical Journal Letters 698 (2009) L142.

[27] A. A. Abdo et al., The Astrophysical Journal 723 (2010) 649.

[28] F. Verechhia et al., in preparation (2011). 